EXTENDED REPORT

\title{
The characterisation of hyalocytes: the origin, phenotype, and turnover
}

\author{
H Qiao, T Hisatomi, K-H Sonoda, S Kura, Y Sassa, S Kinoshita, T Nakamura, T Sakamoto, \\ T Ishibashi
}

Br J Ophthalmol 2005;89:513-517. doi: 10.1136/bjo.2004.050658

See end of article for authors' affiliations

Correspondence to: Koh-Hei Sonoda, MD

PhD, Department of Ophthalmology, Graduate

School of Medical

Sciences, Kyushu

University, 3-1-1

Maidashi, Higashi-ku,

Fukuoka 812-8582

Japan; sonodak@

med.kyushu-u.ac.jp

Accepted for publication

1 September 2004
Aim: To determine the characterisation of hyalocytes: the origin, phenotype, and turnover in the rodent. Methods: To characterise the ultrastructure and distribution of hyalocytes, transmission and scanning electron microscopy was performed in rat eyes. Immunophenotypical analysis was performed by either anti-ED1 or ED2 antibodies. To examine the origin of the hyalocytes, the chimeric mice were created and were used to transplant the bone marrow (BM) cells from enhanced green fluorescent protein (EGFP) transgenic mice. The turnover of hyalocytes was examined at $0,4,6,7$, and 12 months after BM transplantation.

Results: Hyalocytes were distributed especially in the vitreous cortex and had an irregular shape with a spherical granule. Immunophenotypical studies demonstrated that most of the hyalocytes in rat eyes expressed ED2 but not ED1. In the chimeric mice, the hyalocytes were GFP negative right after BM transplantation. Interestingly, more than $60 \%$ of hyalocytes were replaced within 4 months and approximately $90 \%$ within 7 months after BM transplantation.

Conclusions: The rodent hyalocytes were shown to express tissue macrophage marker, were derived from BM, and totally replaced within 7 months. These data provide the characterisation of hyalocytes in physiological conditions, especially their origin, distribution, and turnover, and may contribute to the better understanding of the pathogenesis of vitreoretinal disease.
$\mathrm{T}$ he existence of cells in the peripheral or cortical region of the vitreous body abutting the inner surface of the retina has been described. ${ }^{12}$ These cells, cuurently known as hyalocytes, are located in the vitreous cavity at an average distance of $50 \mu \mathrm{m}$ from the inner surface of the retina and are concentrated anteriorly in the vitreous base and posteriorly in the vicinity of the optic papilla. ${ }^{3}$ Morphological studies have demonstrated that hyalocytes belong to the monocyte/ macrophage lineage but differ significantly from other tissue macrophages. $^{35-8}$ The hyalocytes express monocyte/macrophage cell marker but do not express CD68, glial fibrillary acidic protein (GFAP), cellular retinaldehyde binding protein, and cytokeratin. ${ }^{9-11}$ Although these results may indicate that hyalocyte is derived from monocyte/macrophage but not from retinal cells such as glial cells, and retinal pigment epithelium, there is no definitive evidence of the origin and the regeneration of the hyalocytes. We recently investigated a possible physiopathological role of hyalocytes in eyes. ${ }^{12}$ We demonstrated that hyalocytes could participate not only in proliferative vitreous diseases but also in immunological disorders. However, the characterisation of hyalocytes under physiological or pathological conditions remains unclear.

Recently, enhanced GFP (EGFP) transgenic mice were generated; the tissues of EGFP mice are green under excitation light. ${ }^{13-15}$ GFP fluorescence can easily be detected by flow cytometry or fluorescence microscopy without any cofactor for light emission or any specific staining procedures. ${ }^{16}{ }^{17}$ Experiments using EGFP transgenic mice allow us to track cellular movement in an in vivo model. We thus created chimeric mice by transplanting bone marrow (BM) from EGFP transgenic mice into irradiated wild type (WT) mice. If hyalocyte is derived from haematopoietic progeny, green cells under excitation light will increase in the vitreous cavity of chimeric mice.

In order to investigate the nature of the vitreous in a preliminary study on the morphology, distribution, and immunocytochemical characterisation of hyalocytes within the rodent vitreous, we examined various aspects of hyalocytes. Additionally, the origin and the kinetics of hyalocytes were investigated by using the chimeric mice.

\section{MATERIALS AND METHODS}

\section{Experimental animals}

WT C57BL/6 female mice and Brown Norway rats (postnatal 8 weeks) were obtained from SLC Japan (Shizuoka, Japan). EGFP transgenic female mice (B6 background) ${ }^{15}$ were kindly provided by Dr Masaru Okabe (Osaka University, Japan). All animals were treated humanely and were housed in specific pathogen free conditions at Kyushu University.

\section{Transmission electron microscopy (TEM)}

The rats were anaesthetised with an intraperitoneal injection of pentobarbital and their eyes were enucleated and the posterior segments fixed in $1 \%$ glutaraldehyde and $1 \%$ paraformaldehyde in sterile phosphate buffered saline (PBS). They were then post-fixed in veronal acetate buffer osmium tetroxide $(2 \%)$, dehydrated in ethanol and water, and embedded in Epon. Ultrathin sections were cut from blocks and mounted on copper grids.

\section{Scanning electron microscopy (SEM)}

The removed eye posterior segments were fixed as described in TEM. After dehydration, the retinas were saturated in t-butyl alcohol, and critical point drying was performed (Eiko, Tokyo). The tissue was then placed on stubs by means of self adhesive carbon tabs and sputtered with gold of $20 \mathrm{~nm}$

\footnotetext{
Abbreviations: $B M$, bone marrow; EGFP, enhanced green fluorescent protein; FACS, flow cytometry analysis; GFAP, glial fibrillary acidic protein; ILM, inner limiting membrane; PBS, phosphate buffered saline; $\mathrm{PI}$, propidium iodide; SEM, scanning electron microscopy; TEM, transmission electron microscopy
} 

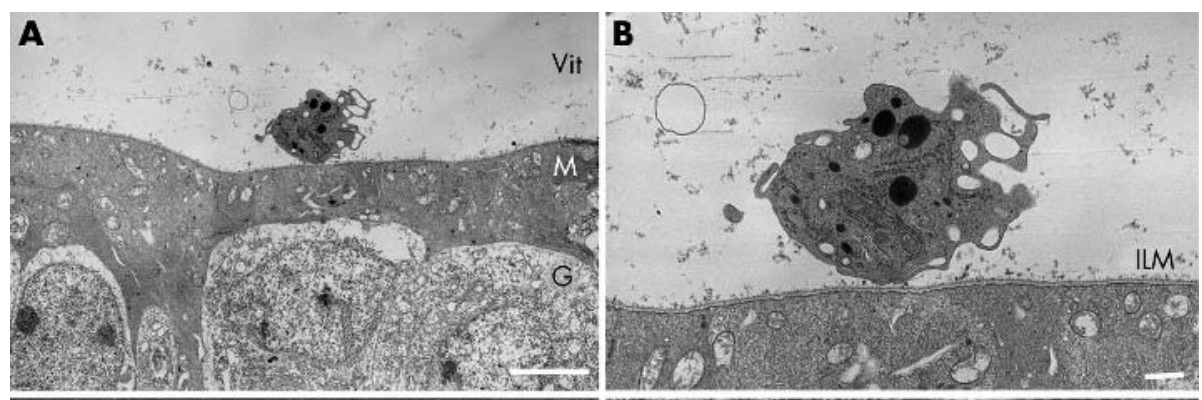

Figure 1 The morphological characteristics and the distribution of hyalocytes. TEM micrograph of a hyalocyte was distributed in the vitreous cavity close to the retina (A). Higher magnification showed the hyalocyte was completely free and close to the inner limiting membrane (ILM) of the retina (B). SEM micrograph showed three of the free hyalocytes were distributed randomly in the vitreous cortex very close to the retina, which lies in the background (C). A higher
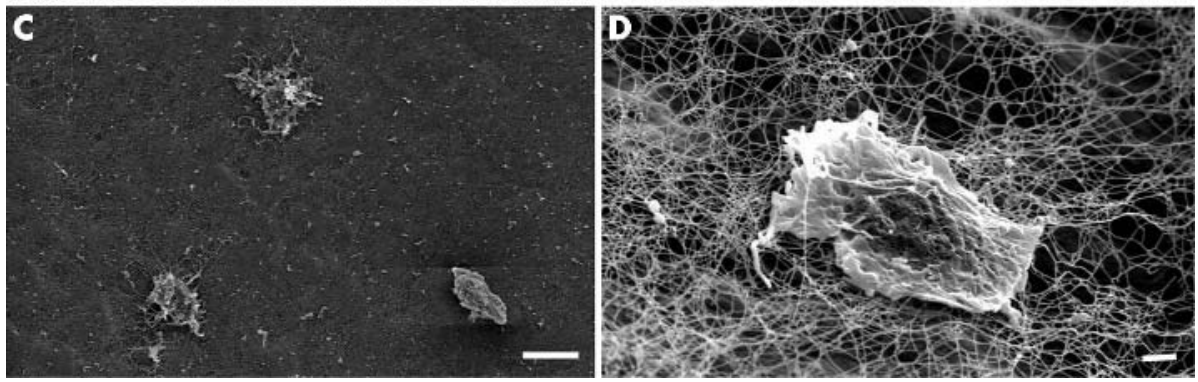
magnification view showed the cell was entangled in a collagen fibril network in the vitreous cortex and a few protuberances are observed at the cell surface (D). (Original magnification A, $\times 2600$, bar $5 \mu \mathrm{m} ; \mathrm{B}, \times 6000$, bar $1 \mu \mathrm{m} ; \mathrm{C}, \times 1100$, bar $10 \mu \mathrm{m} ; \mathrm{D}$, $\times 4300$, bar $=1 \mu \mathrm{m})$.

thickness by argon plasma coater (Eiko). Next, the retina was studied using a Jem 840 scanning electron microscope (Jeol).

\section{Immunohistochemistry}

For immunohistochemical examinations, the rat eyes were enucleated and fixed in $4 \%$ paraformaldehyde, embedded in paraffin. Anti-ED1, ED2 antibodies (Serotec, Oxford, UK), and control IgG were used at a 1:100 dilution. Anti-EDl antibody recognises monocyte macrophage, and anti-ED2 recognises tissue macrophages in rat. ${ }^{18}$ Propidium iodide (PI) was used for nuclear staining.

Cy5 labelled secondary antibody (Zymed Laboratories, San Francisco, CA, USA) was used at a dilution of 1:200 for 20 minutes, and then observed by fluorescence microscopy (Olympus, Tokyo).

\section{Preparation of cell suspensions and chimeric mice}

To characterise the infiltrating hyalocytes, we produced chimeric GFP mice, by a previously described method. ${ }^{19}$ Briefly, the WT B6 mice were lethally irradiated with $\mathrm{x}$ ray of $9 \mathrm{~Gy}$; these eyes were protected with lead shields to prevent radiation retinopathy. Then they were intravenously injected via a caudal vein with $0.2 \mathrm{ml}$ of PBS containing EGFP transgenic mice BM cells $\left(6 \times 10^{6}-1 \times 10^{7}\right)$. Successful BM transplantation was confirmed by the identification of $\mathrm{GFP}^{+}$cells in the blood 2 weeks after irradiation and then used for experiments.

\section{In vivo cellular tracking using fluorescence microscopy}

To directly observe the donor derived $\mathrm{GFP}^{+}$cells, the peripheral blood cells in chimeric mice were obtained and suspended in $2 \mu \mathrm{g} / \mathrm{ml}$ PI for identification of the nuclei in cells, they were then observed by fluorescence microscopy (Olympus, Tokyo). The eyes from WT mice were used as control eyes.

\section{Flow cytometry analysis (FACS)}

The spleen cells of the chimeric mice were obtained and determined by flow cytometry using EPICS XL (Becman Coulter, Mannheim, Germany). GFP fluorescence was measured at the same excitation/emission wavelength as FITC.

\section{Histological analysis}

The chimeric mice were sacrificed at $0,4,6,7$, and 12 months after BM transplantation. The eyes were enucleated, futher
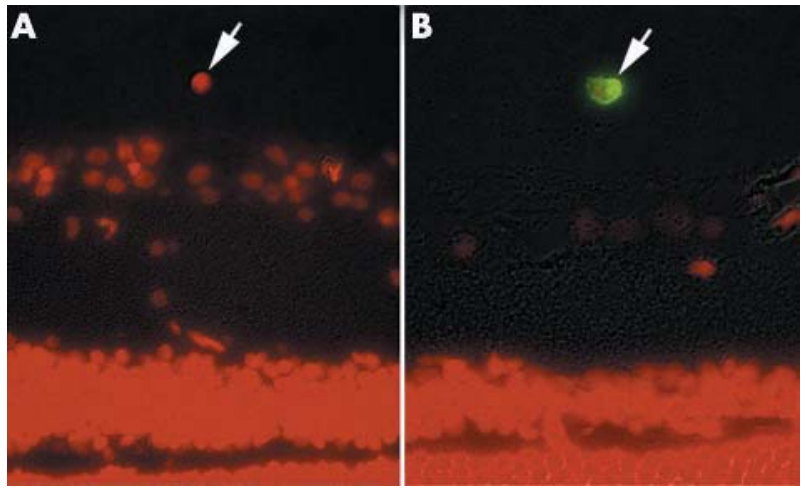

Figure 2 Immunophenotypic analysis of hyalocytes characterisation. The normal adult rat hyalocytes were double immunostained with EDI and $\mathrm{PI}, \mathrm{ED} 2$ and $\mathrm{PI}$, respectively at 6 months post-transplantation. ED1 antibody, bearing the characteristic phenotype of monocyte derived macrophages, did not react with hyalocytes $\left(\mathrm{Pl}^{+}, \mathrm{EDI}^{-}\right)(\mathrm{A}$, arrowhead) The ED2 expression was typically associated with hyalocytes $\left(\mathrm{PI}^{+}, \mathrm{ED} 2^{+}\right)$ $(B$, arrowhead). (Original magnification $\times 400)$.

fixed in 2\% paraformaldehyde for 3-4 hours and dehydrated in acetone for 5 minutes at $4^{\circ} \mathrm{C}$, embedded in Technovit 8100 (Heraeus Kulzer, Werheim, Germany) on crushed ice and cut to $3 \mu \mathrm{m}$ thick serial sections. The samples were observed by fluorescence microscopy (Olympus, Tokyo).

\section{Statistical analysis}

The number of cells per $1 \mathrm{~mm}$ area in 10 histological sections for each eye was measured and six eyes were analysed using analysis software (MacScope, Mitani, Fukui, Japan). The results were expressed as the means (SD). Student's $t$ test was used to calculate the probability by comparing data between the groups and $\mathrm{p}<0.05$ was considered to be significant.

\section{RESULT}

\section{Hyalocytes}

Hyalocytes were examined in rat vitreous cavity by TEM and SEM. Ultrastructural characterisation of the rat hyalocytes was performed using the electron microscope (fig l).

Figure 1A illustrated a TEM micrograph of a hyalocyte distributed in the vitreous cavity close to the retina. The cell 

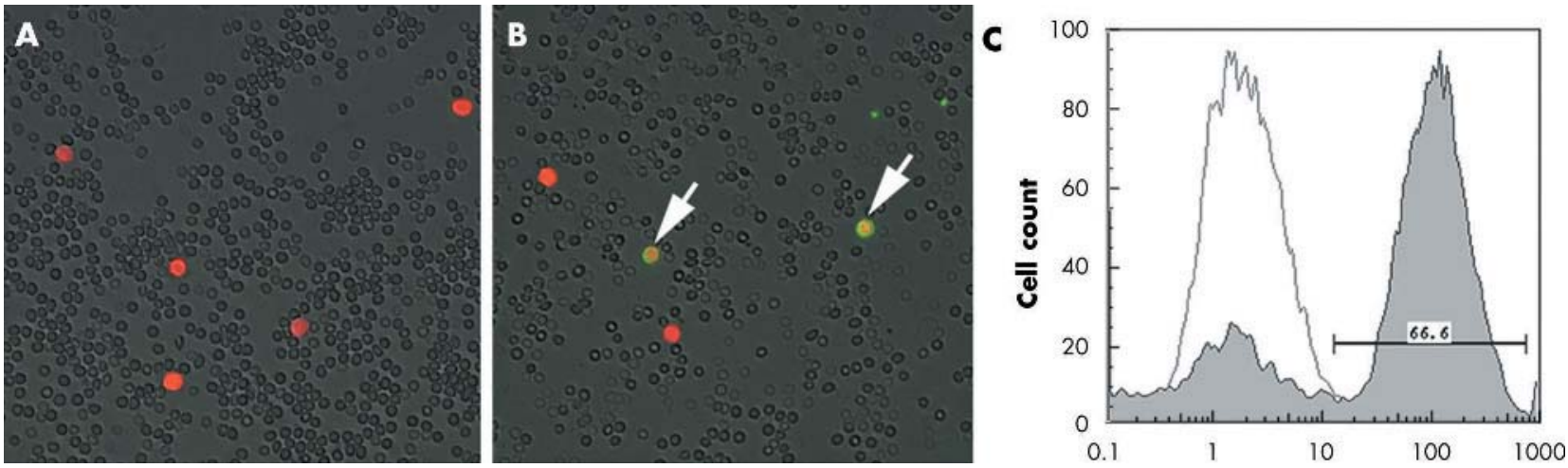

Figure 3 Fluorescein microscope analyses of peripheral leucocytes in chimeric mice. Four weeks after reconstitution, the peripheral blood leucocytes of normal C57BL/6 as a control (A). In the chimeric mice, some of the peripheral leucocytes expressed both $\mathrm{Pl}^{+}$and GFP+ (yellow) (B). (Original magnification $\times 100)$. Flow cytometric analyses of splenocyte chimerisms of radiation BM chimeras (C).

surface was irregular with numerous slender processes. Higher magnification of the micrograph (fig 1B) clearly showed the hyalocyte was a completely free cell separated from the inner limiting membrane (ILM) of the retina. It was surrounded by collagen fibrils in the vitreous cortex. Numerous electron dense heterogeneous contents were observed inside the hyalocyte. The cytoplasmic granules showed the morphological characteristics of lysosomes: a single membrane, a halo, and a dense homogeneous matrix.

The examination of the hyalocytes with the SEM has previously been described in the rabbit. ${ }^{20}$ Here, we examined them in the rat. The hyalocytes revealed their complex and warty surface. In a survey view of the posterior part of the eyeball, free hyalocytes were found distributed randomly on the vitreous cortex of the retinal ILM (fig IC). A high magnification view of the hyalocyte showed the cell was entangled in a collagen fibril network in the vitreous cortex (fig 1D). The same feature of hyalocytes was also observed in mice (data not shown).

\section{Immunophenotypic analysis of hyalocytes expressing ED2}

Immunohistochemical examinations were performed to analyse and characterise the rat vitreous hyalocytes. The adult rat hyalocytes were double immunostained with EDI and PI, ED2 and PI, respectively. EDI recognises an antigen in monocytes and in most macrophages, free and fixed. ${ }^{21}$ ED2 recognises membrane and cytoplasmic antigens of tissue macrophages, discriminating between distinct subpopulations of macrophages. ${ }^{21}$ The immunophenotypic analysis demonstrated that most of the hyalocytes were ED2 positive (90\%), and few hyalocytes were EDl positive (15\%). Only 5\% of hyalocyte showed both ED1 and ED2 positive staining. Antibody directed against EDl, bearing the characteristic phenotype of monocyte derived macrophages, did not react with hyalocytes (fig 2A). Antibody directed against ED2 was typically associated with hyalocytes, and illustrated that vitreous hyalocytes have the characteristic phenotype of tissue macrophages (fig 2B).

\section{GFP $^{+}$cells detected in BM chimeras}

Four weeks after reconstitution, the peripheral blood cells and the splenocytes from the radiation BM chimeric mice were examined for GFP expression. The green light of the native GFP or fusion proteins can be detected by fluorescence microscopy or flow cytometry in living cells without fixation. ${ }^{15}$ As a control, the peripheral leucocytes of the normal C57BL/6 mouse observed $\mathrm{PI}^{+}$by a fluorescein microscope (fig 3A). In contrast, in the radiation BM chimeric mice, some of the peripheral leucocytes expressed both $\mathrm{PI}^{+}$and $\mathrm{GFP}^{+}$(fig 3B). Moreover, we also obtained the chimeric mice splenocytes and analysed them by flow cytometry. After 4 weeks of BM transplatation, approximately $66 \%$ of splenocytes from chimeras contained a high density of $\mathrm{GFP}^{+}$(fig 3C).

\section{Hyalocytes regenerated and totally replaced within 7 months}

To further elucidate the origin and regeneration of hyalocytes, we also examined the chimeric mice at $0,4,6,7$, and 12 months after BM transplatation. As determined microscopically, 0 day after BM transplantation, the hyalocytes contained only PI but not GFP in vitreous cavity (fig 4A). Virtual results were observed in naive mouse vitreous (data not shown). Six months after BM transplantation, the donor derived $\left(\mathrm{GFP}^{+}\right)$hyalocytes were readily observed in the chimeric vitreous cavity (fig 4B). Moreover, the $\mathrm{GFP}^{+}$ hyalocytes were distributed in the vitreous cavity close to the retina as described above (fig 1). These results suggested that hyalocytes are derived from BM. By evaluating the average ratios of $\mathrm{GFP}^{+}$cells in total $\mathrm{PI}^{+}$cells in vitreous cavity, we examined the turnover period of the hyalocytes. The hyalocytes were replaced gradually after BM cells transplantation. Four months after BM transplantation, more than $60 \%$ of hyalocytes were replaced by the BM derived $\mathrm{GFP}^{+}$ cells. Approximately $90 \%$ of total vitreous hyalocytes were $\mathrm{GFP}^{+}$in the chimeric mice 7 months after BM transplantation (fig 4C). These cells have a similar character and distribution to the naive hyalocytes by TEM and SEM (data not shown). Taken together, these results indicate that the rodent hyalocytes were of $\mathrm{BM}$ origin and regenerate gradually even under the physiological conditions.

\section{DISCUSSION}

We demonstrated for the first time that rodent hyalocytes were derived from BM cells. Hyalocytes were found in the vitreous cavity and most of the hyalocytes had the morphological characteristics of macrophage that expressed ED2, but not ED1, confirming their identification as tissue macrophages. The same immunophenotypical characterisation was also observed in murine hyalocytes by F4/80 (data not shown). By using the chimeric mice, we revealed that the $\mathrm{BM}$ derived hyalocytes $\left(\mathrm{GFP}^{+} / \mathrm{PI}^{+}\right)$were turned over gradually, and replaced almost all of the hyalocytes in 6 months These results suggest that BM derived cells might differentiate into hyalocytes and turn over even under normal physiological conditions. The large number of BM derived cells lining in the vitreous cavity suggests that they make a contribution to maintaining the vitreous. 

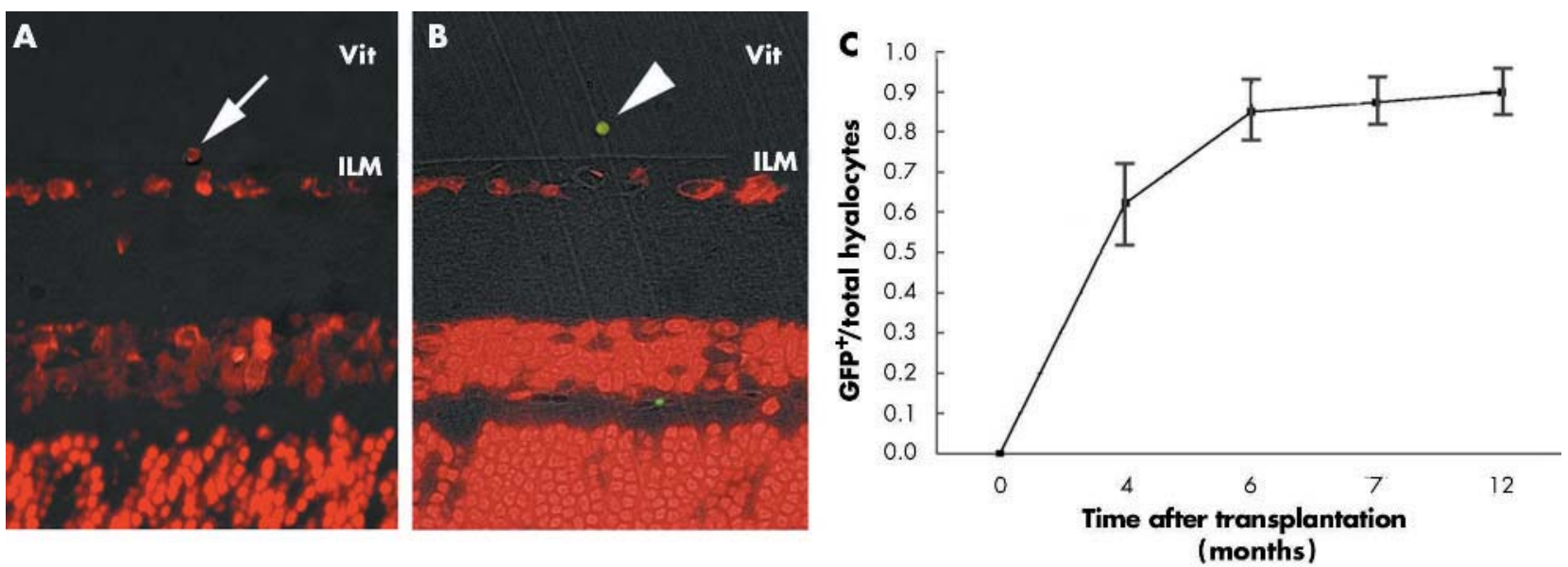

Figure 4 The kinetics of the hyalocytes. Chimeric mice were used to analyse the hyalocytes' origin and regeneration at 0,4, 6, 7, and 12 months after $\mathrm{BM}$ transplantation by fluorescent microscopy. The $\mathrm{Pl}^{+}$nuclei associated $\mathrm{GFP}^{-}$cells, indicating recipient derived hyalocyte, were seen in the mouse right after BM transplantation (A). The donor derived (GFP') hyalocytes were observed 6 months after BM transplantation (B). The time dependent ratio of $\mathrm{GFP}^{+}$hyalocytes in the chimeric mice $(C)$ (original magnification $\left.\times 400\right)$.

We also indicated that $\mathrm{BM}$ derived $\mathrm{GFP}^{+}$cells $\left(\mathrm{GFP}^{+} / \mathrm{PI}^{+}\right)$ occurred and turned over the residual host hyalocytes in the vitreous cavity with time. The kinetics of both resident and infiltrating macrophages have been thoroughly investigated in brain by GFP chimeric models. ${ }^{22}$ The turnover of resident macrophages, however, was not revealed adequately. Several studies revealed that the BM derived cells infiltrated into the central nerve system (CNS) across the blood-brain barrier in normal condition. ${ }^{23} 24$ Although infiltrating/GFP ${ }^{+}$macrophages were physiologically (without any injury or inflammation) detected in the brain of the chimeric mouse, the proportion of $\mathrm{GFP}^{+}$macrophages was small. ${ }^{22}{ }^{25}$ Even under the pathological coditions including demyelation and ischaemic injury, resident/GFP ${ }^{-}$macrophages were dominant compared with $\mathrm{GFP}^{+}$macrophages for up to 4 weeks. ${ }^{26}$ In contrast, we revealed that $90 \%$ of resident macrophages in vitreous cavity were physiologically replaced by BM derived macrophages for up to 7 months. Our data suggested that levels of resident macrophages might not been maintained by their proliferation but by being produced by BM in physiological situations. This discrepancy between brains and eyes remains unclear. The observation period might be one of the reasons for the discrepancy between replacement rates of tissue macrophages (CNS and retinas). In these studies, many $\mathrm{GFP}^{+}$cells existed around blood vessels in the CNS. ${ }^{22-26}$ Hyalocytes examined in this study exist directly on the ILM near the vascular rich region. The location might be another possible reason of this discrepancy. Further studies should be done to check whether tissue specific mechanisms, which make circulating macrophages infiltrate and reside in the tissue, exist.

Chimeric mice stably reconstituted with BM cells represent a good model for analysis of the mechanism of BM cell infiltration in the eye. However, in preparing chimeric mice, irradiation of the recipient mice is necessary to kill their own $\mathrm{BM}$ before transplantation; this might induce an inflammatory response. This irradiation causes gliosis by activation of astrocytes and microglia in the brain. ${ }^{22}{ }^{23}$ We used a lead cup to prevent the eyes being damaged; we did not detect any histological changes such as gliosis and acute inflammatory responses in all retinas examined.

Although our data cannot definitively conclude a role for hyalocytes in the vitreous cavity, the inhibitory effects of vitreous hyalocytes on endothelial cell and RPE proliferation have been recently demonstrated..$^{10}{ }^{11}$ Further investigations are needed to delineate the functional characterisation of hyalocytes. Increased knowledge of the origin and character of hyalocytes should improve our understanding of various vitreoretinal pathologies, such as epiretinal membrane formation, proliferative vitreoretinopathy, and diabetic retinopathy, and lead to improved methods of prevention; furthermore, this will be also an important step towards optimising regenerative therapies.

\section{Authors' affiliations}

H Qiao, T Hisatomi, K-H Sonoda, Y Sassa, T Ishibashi, Department of Ophthalmology, Graduate School of Medical Sciences, Kyushu University, Fukuoka, Japan

S Kura, Department of Medical Biophysics and Radiation Biology, Graduate School of Medical Sciences, Kyushu University, Fukuoka, Japan

S Kinoshita, T Nakamura, Department of Ophthalmology, Kyoto Prefectural University of Medicine, Kyoto, Japan

T Sakamoto, Department of Ophthalmology, Graduate School of Medical Sciences, Kagoshima University, Kagoshima, Japan

\section{REFERENCES}

1 Szirimai JA, Balazs EA. Studies on the structure of the vitreous body. III. Cells in the cortical layer. AMA Arch Ophthalmol 1958;59:34-48.

2 Hamburg A. Some investigations on the cells of the vitreous body. Ophthalmologica 1959;138:81-107.

3 Balazs EA, Toth LZJ, Eeckl EA, et al. Studies on the structure of the vitreous body. XII. Cytological and histochemical studies on the cortical tissue layar. Exp Eye Res 1964;3:57-71.

4 Sebag J. The vitreous. In: Hart WM Jr, ed. Adler's physiology of the eye. 9th ed. St Louis: Mosby Year Book, 1992:268-347

5 Balazs EA, Toth LZ, Ozanics V. Cytological studies on the developing vitreous as related to the hyaloid vessel system. Graefes Arch Clin Exp Ophthalmol 1980;213:71-85.

6 Saga T, Tagawa Y, Takeuchi T, et al. Electron microscopic study of cells in vitreous of guinea pig. Jpn J Ophthalmol 1984;28:239-47.

7 Salu P, Claeskens W, De Wilde A, et al. Light and electron microscopic studies of the rat hyalocyte after perfusion fixation. Ophthalmic Res 1985;17:125-30.

8 Ogawa K. Scanning electron microscopic study of hyalocytes in the guinea pig eye. Arch Histol Cytol 2002;65:263-8

9 Lazarus HS, Hageman GS. In situ characterization of the human hyalocyte. Arch Ophthalmol 1994;112:1356-62.

10 Schonfeld CL. Hyalocytes inhibit retinal pigment epithelium cell proliferation in vitro. Ger J Ophthalmol 1996;5:224-8.

11 Jacobson B, Dorfman T, Basu PK, et al. Inhibition of vascular endothelial cell growth and trypsin activity by vitreous. Exp Eye Res 1985;41:581-95.

12 Sakamoto T. Cell biology of hyalocytes. Nippon Ganka Gakkai Zasshi 2003; 107:866-82.

13 Chalfie M, Tu Y, Euskirchen G, et al. Green fluorescent protein as a marker for gene expression. Science 1994;263:802-5.

14 Cubitt $A B$, Heim R, Adams SR, et al. Understanding, improving and using green fluorescent proteins. Trends Biochem Sci 1995;20:448-55.

15 Okabe M, Ikawa M, Kominami K, et al. "Green mice" as a source of ubiquitous green cells. FEBS Lett 1997;407:3131-9. 
16 Chalfie M, Tu Y, Euskirchen G, et al. Green fluorescent protein as a marker for gene expression. Science 1994;263:802-5.

17 Kawakami N, Nishizawa F, Sakane N, et al. Roles of integrins and CD44 on the adhesion and migration of fetal liver cells to the thymus. J Immunol 1999:163:3211-16.

18 Dijkstra CD, Dopp EA, Joling P, et al. The heterogeneity of mononuclear phagocytes in lymphoid organs: distinct macrophage subpopulations in the rat recognized by monoclonal antibodies ED1, ED2 and ED3. Immunology 1985;54:589-99.

19 Hisatomi T, Sakamoto T, Sonoda KH, et al. Clearance of apoptotic photoreceptors: elimination of apoptotic debris into the subretinal space and macrophage-mediated phagocytosis via phosphatidylserine receptor and integrin alphavbeta3. Am J Pathol 2003:162:1869-79

20 Haddad A, Andre JC. Hyalocyte-like cells are more numerous in the posterior chamber than they are in the vitreous of the rabbit eye. Exp Eye Res 1998;66:709-18
21 Masuda H, Asahara T. Post-natal endothelial progenitor cells for neovascularization in tissue regeneration. Cardiovasc Res 2003;58:390-8.

22 McMahon EJ, Suzuki K, Matsushima GK. Peripheral macrophage recruitment in cuprizone-induced CNS demyelination despite an intact blood-brain barrier. J Neuroimmunol 2002;130:32-45.

23 Eglitis MA, Mezey E. Hematopoietic cells differentiate into both microglia and macroglia in the brains of adult mice. P Natl Acad Sci 1997;94:4080-5.

24 Bush TG, Puvanachandra N, Horner $\mathrm{CH}$, et al. Leukocyte infiltration, neuronal degeneration, and neurite outgrowth after ablation of scar-forming, reactive astrocyłes in adult transgenic mice. Neuron 1999;23:297-308.

25 Mueller M, Leonhard C, Wacker K, et al. Macrophage response to peripheral nerve injury: the quantitative contribution of resident and hematogenous macrophages. Lab Invest 2003;83:175-85.

26 Schilling M, Besselmann M, Leonhard C, et al. Microglial activation precedes and predominates over macrophage infiltration in transient focal cerebral ischemia: a study in green fluorescent protein transgenic bone marrow chimeric mice. Exp Neurol 2003;183:25-33. 